\title{
Exploring Regimes in Black Hole Scaling
}

\author{
Sebastian Heinz ${ }^{1} \dagger$, and Andrea Merloni ${ }^{2}$ \\ ${ }^{1}$ University of Wisconsin-Mdiosn, \\ Madison, WI 53706, USA \\ email: heinzs@astro.wisc.edu \\ ${ }^{2}$ Max-Planck-Institute for Extraterrestrial Physics \\ Garching, Germany \\ email: am@mpe.mpg.de
}

\begin{abstract}
Recent observational evidence suggests the existence of two tracks in the radio-Xray relation for X-ray binaries. Claims have also been made for deviations from the so-called fundamental plane of black hole activity due to the influence of radiative cooling on synchrotron emission from jets and the relative importance of disk and jet emission. In addition, cases of strongly boosted classes of objects, such as BL Lacs, show evidence for jet emission in their location relative to the fundamental plane. In light of the recent literature activity discussing these issues, we revisit the scaling relations expected for synchrotron emission from jet cores. We review the set of scaling laws expected for different types of emission and discuss their relevance to the new observational data, and the conditions under which breaks in the observed scaling relations should be expected. None of the canonical cases offer a satisfactory explanation for the best fit slope of the steep branch of the radio-X-ray relation in hard-state X-ray binaries.
\end{abstract}

Keywords. Jets, Synchrotron Emission, Black Holes

\section{Introduction: Scale Invariance}

The underlying assumption in attempting to connect jet emission over a large range in accretion rate and black hole mass is that jet physics is essentially self-similar and that it is reasonable to assume that jets behave in a scale invariant fashion when changing black hole mass and accretion rate. This is well motivated for black holes accreting in a state in which energy transport is dominated by mechanical processes, in which case the accretion solution itself can be expected to be scale invariant in mass, and, to lowest order, in accretion rate (global accretion simulations, which by and large have been possible only in such a mechanically cooled state, suggest that this is the indeed case).

Mathematically, any relevant dynamical quantity in a scale invariant jet can be separated into the product of two functions $\phi(M, \dot{m}, a) \psi\left(\vec{r} / r_{\mathrm{g}}, a\right)$, where $r_{\mathrm{G}}=G M / c^{2}$ and $a$ is the black hole spin parameter.. We refer to this decomposition as the scale invariance condition, and in the following, we will assume that the important functional dependences of the magnetic field and particle pressure can be represented as $\phi_{\mathrm{B}} \propto \sqrt{\dot{m} / M}$ and $\phi_{\mathrm{p}} \propto \dot{m} / M$, appropriate for a wide range of accretion solutions (and in particular, mechanically cooled accretion flows, such as ADAF and ADIOS solutions).

Heinz \& Sunyaev (2003) and Heinz (2004) showed that any jet model that satisfies the scale invariance condition must obey very generic scaling relations for its synchrotron emission. These scaling laws are simply expressions of the properties of self-absorbed synchrotron radiation for powerlaw distributions of electrons. For the canonical case of synchrotron emission not affected by radiative cooling for an electron distribution of

$\dagger$ This work was in part supported through grant AST1109347 from the National Science Foundation 
index $p=2$, the luminosity must follow the proportionality

$$
L_{\nu} \propto M^{\frac{17-4 \alpha}{12}} \dot{m}^{\frac{17+8 \alpha}{12}}
$$

while for optically thin radiation subject to the effects of synchrotron cooling, the luminosity of the jet must follow

$$
L_{\nu} \propto M^{\frac{6-\alpha}{4}} \dot{m}^{\frac{10-\alpha}{4}}
$$

where $\alpha$ is the spectral index, defined as $\alpha \equiv-\partial \log L_{\nu} / \partial \log n u$. Here and in the following, we define the accretion rate in terms of the Eddington rate for a given mass, $\dot{m} \equiv \dot{M} / M_{\mathrm{Edd}}$.

These relations apply to the core emission of jets from accreting black holes, for which the effects of interaction with the external medium can be neglected.

It is worth pointing out again here that the scaling relation between jet luminosity and black hole mass is of fundamental nature, given that scale invariance of the metric is guaranteed and that purely mechanically cooled accretion solutions can be expected to be strictly scale invariant. In some cases, the literature refers to this scaling relation as a "correction" to an underlying radio-X-ray relation [e.g., Broderick, \& Fender (2011)]. However, given its more fundamental nature, this wording is misleading and we suggest avoiding such terminology.

In order to test these predicted scaling relations, we must find a way to measure or estimate the mass, accretion rate, and the jet luminosity in a given band. The easiest way to identify and measure emission from the jet is in the radio band, where synchrotron emission typically dominates thermal emission from the accretion flow itself. While effects of relativistic beaming will affect the radio emission from a jet, Heinz \& Merloni (2004) showed that it is reasonable to expect that, in a large enough sample selected in bands other than the radio, relativistic beaming will affect the observations in a relatively benign manner.

Black hole mass measurements are possible through a number of means as well (a review of black hole mass measurement techniques is beyond the scope of this manuscript).

However, the accretion rate is not directly measurable and it will be necessary to formulate observational proxis. It is this process that introduces ambiguities into tests of scale invariance.

In the recent literature, the $\mathrm{X}$-ray luminosity has been used as a proxi for the accretion rate to search for observational evidence of relations between jet emission and the underlying black hole parameters. This has led to the discovery of the radio-X-ray relation by Gallo, Fender, \& Pooley (2003) and the fundamental plane of black hole activity first discussed in Merloni, Heinz, \& di Matteo (2003) and Falcke, Körding, \& Markoff (2004).

As might be expected from the fact that scale invariance is only expected to hold faithfully in mechanically cooled low-accretion rate states (such as ADAF solutions), these relations are restricted to X-ray binaries in the hard state and low-luminosity AGN. Below, we will discuss these relations in more detail.

If the X-ray luminosity is interpreted as coming from a raditively inefficient accretion flow (i.e., one in which the energy transport is mechanical), the X-ray emission can be expected to be proportional roughly to the square of the accretion rate. In this case, it has been shown that the correlation coefficients of the radio-X-ray relation and the fundamental plane are consistent with the scale invariance predictions.

Alternatively, is has been proposed that the X-ray emission of a large fraction of sources in the samples is dominated by emission from the jet (either synchrotron or inverse Compton emission). While the correlation coefficients predicted from scaling relations in this case differ somewhat from those in the accretion dominated case, it has been difficult 
to differentiate between the two cases observationally [Heinz (2004)]. Thus, whether the $\mathrm{X}$-ray emission is, in fact, stemming from the accretion flow itself or originating in the jet has been hotly debated and is far from settled.

Only in cases where the jet emission is strongly boosted towards the observer can we be confident that the X-ray emission is dominated by the jet (such as in BL-Lac objects and blazars). For this reason, the original fundamental plane sample was specifically selected to exclude such objects. From the point of view of testing scale invariance predictions, this is a prudent path to take, since a sample dominated by X-ray emission from jets would not contain independent information about $\dot{m}$ (the radio-X-ray relation would purely be an expression of the jet spectrum). See Plotkin, Markoff, Kelly, Körding, \& Anderson (2012) for a discussion focussed on strongly boosted objects.

It is also clear that, in the case of X-ray binaries, the X-ray emission in some accretion states is dominated by a standard thin disk (evidenced by thermal emission in the soft state). Objects in the soft state display heterogeneous radio properties and it is generally believed that most of these objects are radio quiet, while sources in the hard state are uniformly radio loud in the sense that they display radio emission.

From the hardness-intensity diagram in XRBs, it is clear that transitions between soft and hard state happen over a range of X-ray luminosities and thus accretion rates. A one-to-one mapping between X-ray luminosity and radio loudness state is thus not possible. In the discussion below, we will arbitrarily assume that this transition happens at accretion rates between $10^{-3} \leqslant \dot{m} \leqslant 10^{-1}$ and, rather than treating is stochastically, we will further assume a fixed transition rate of $\dot{m}_{\mathrm{crit}}=10^{-2}$ for simplicity.

Given that accretion rate changes and relativistic boosting can alter the X-ray emission mechanisms and the relative intensity of disk and jet emission, and given that radiative effects like synchrotron cooling and optical depth vary with accretion rate and mass, it is clear that we cannot expect a single scaling relation to hold for all jets in the hard state at all accretion rates and black hole masses.

This was pointed out already in Heinz (2004) and subsequently explored in one limit by Yuan, Yu, \& Ho (2009). The recent discovery of an alternative track in the radio$\mathrm{X}$-ray relation for $\mathrm{X}$-ray binaries, the intrinsic scatter that has always been present in realizations of the fundamental plane, and the discussion of strongly boosted sources in recent work by Plotkin, Markoff, Kelly, Körding, \& Anderson (2012) further indicate that deviations from a single scaling should be expected. Motivated by this argument, we will now review the different scaling regimes to be expected over a range in black hole mass and accretion rate in generic terms.

\section{Scaling Regimes}

We can categorize different regimes in the black hole scaling relations by the origins of their radio and X-ray emission. In this work, we distinguish two cases of radio synchrotron emission, both of which follow the scaling $L_{\mathrm{R}} \propto M^{\frac{13+2 p-(p+2) \alpha}{2(p+4)}} \dot{m}^{\frac{13+2 p+(p+6) \alpha}{2(p+4)}}$ derived in Heinz \& Sunyaev (2003), where $p \approx 2$ is the electron spectral index and alpha is the synchrotron spectral index:

- Optically thick emission with $L_{\mathrm{R}} \propto M^{17 / 12} \dot{m}^{17 / 12}$

- Optically thin emission for large mass and low jet power, with $L_{\mathrm{R}} \propto M^{5 / 4} \dot{m}^{7 / 4}$.

We distinguish two cases of disk and jet emission each for the source of the X-rays. In the case of emission by the accretion flow, we simply parameterize the dependence on accretion and mass as $L_{\mathrm{X}} \propto M \dot{m}^{\zeta}$, following Merloni, Heinz, \& di Matteo (2003). In 


\begin{tabular}{|c|c|c|c|c|c|c|c|}
\hline \multicolumn{2}{|c|}{ | Radio |X-ray } & \multirow{2}{*}{$\begin{array}{l}\mid \xi_{\mathrm{RM}} \\
\mid \underline{(2 p+13)\left(\zeta_{\mathrm{A}}-1\right)-\alpha\left[\zeta_{\mathrm{A}}(p+2)+p+6\right]}\end{array}$} & | $\xi_{\mathrm{RM}, \mathrm{fid}}$ & \multicolumn{2}{|c|}{\begin{tabular}{l|l} 
Radio | X-ray \\
\end{tabular}} & $\xi_{\mathrm{RX}}$ & $\overline{\mid} \xi_{\mathrm{RX}, \mathrm{fid}}$ \\
\hline thick & $\mathrm{ADAF}$ & & $\approx 0.8$ & thick & ADAF & $\frac{2 p+13+(p+6) \alpha}{2(p+4)}$ & $\approx 0.62$ \\
\hline thick & disk & $2 \zeta_{\mathrm{A}}(p+4)$ & $\approx 0$ & thick & disk & $2 p+13+(p+6) \alpha$ & $\approx 1.42$ \\
\hline thick & thin jet & $\frac{(2 p+13)(p-1-2 \alpha)}{(p+4)(p+5)}$ & $\approx 0.4$ & thick & thin jet & $2 \frac{2 p+13+(p+6) \alpha}{2}$ & $\approx 0.8$ \\
\hline thick & cooled jet & $\frac{(2 p+13)(p-1-2 \Delta-2 \alpha)-4 \alpha \Delta(p+1)}{2(p+4)(p+5-6 \Delta)}$ & $\approx 0$ & thick & cooled jet & $\frac{2 p+13+(p+6) \alpha}{2 p+13+(p+6) p / 2}$ & $\approx 1.42$ \\
\hline thin & $\mathrm{ADAF}$ & $\frac{7-p}{4}-\frac{5+p}{4 \zeta_{A}}$ & $\approx 0.49$ & thin & ADAF & $\frac{5+p}{4 \zeta}$ & $\approx 0.76$ \\
\hline thir & disk & $\frac{1-p}{2}$ & $\approx-0.5$ & thin & di & $\frac{5+p}{4}$ & $\approx 1.75$ \\
\hline this & thin jet & & $=0$ & thin & thin jet & $1^{4}$ & $=1$ \\
\hline thin & cooled jet & $\mid \frac{(2 p-8) \Delta}{5+p-6 \Delta}$ & $\approx-0.5$ & thin & cooled jet & $\frac{p+5}{5+p-6 \Delta}$ & $\approx 1.75$ \\
\hline
\end{tabular}

Table 1. Table of scaling indices for different radio and X-ray emission mechanisms, as functions of the electron powerlaw spectral index $p$, the observed radio spectral index $\alpha$, the X-ray efficiency index $\zeta_{\mathrm{A}}$, and the cooling break $\Delta$ in cases of X-ray emission from electrons affected by synchrotron cooling. The fiducial values for the canonical case of $p=2, \alpha=0, \zeta_{\mathrm{A}}=2.3$, and $\Delta=0.5$ are shown in grey.

the case of synchrotron emission, we consider optically thin emission of either cooled or uncooled electrons, assuming a classic broken powerlaw with a $\Delta \alpha \approx 0.5$ in the case of cooling. In this case, we can use the scaling relations derived by Heinz (2004), which give $L_{\mathrm{X}} \propto M^{\frac{7-p-2 \Delta}{4}} \dot{m}^{\frac{5+p-6 \Delta}{4}}$. The four resulting scaling regimes are

- Efficient disk emission for high accretion rates, with $L_{\mathrm{X}} \propto M \dot{m}$

- Inefficient accretion emission for low rates with $L_{\mathrm{X}} \propto M \dot{m}^{2.3}$

- Opticaly thin synchrotron emission from un-cooled electrons with $L_{\mathrm{X}} \propto M^{5 / 4} \dot{m}^{7 / 4}$.

- Cooled synchrotron emission with $L_{\mathrm{X}} \propto M \dot{m}$.

The scaling relations between $L_{\mathrm{R}}, L_{\mathrm{X}}$, and $M$ are readily derived from the scaling relations above by substituting the X-ray luminosity for the accretion rate similar to Merloni, Heinz, \& di Matteo (2003). The eight possible combinations of the scaling indeces are listed in table 1.

It is important to note that the transitions from optically thick to thin emission, and from synchrotron cooled to un-cooled emission, occur at characteristic frequencies $\nu_{\mathrm{br}}$ and $\nu_{\text {cool }}$ respectively, which depend on $\dot{m}$ and $M$. The numerical value of the transition frequencies depends on the specifics of the jet model, but the scaling behavior of both frequencies with black hole mass and accretion rate can be explicitly evaluated through their dependence on B-field, particle pressure, and characteristic length scale (which themselves can be expected to follow the canonical scalings with mass and accretion rate in mechanically cooled flows such that $\left.\phi_{\mathrm{B}}^{2} \propto \phi_{\mathrm{p}} \propto \dot{m} / M\right)$. Then,

$$
\nu_{\mathrm{br}} \propto\left(M \phi_{\mathrm{p}}\right)^{\frac{2}{p+4}} \phi_{\mathrm{B}}^{\frac{p+2}{p+4}} \propto M^{-1 / 3} \dot{m}^{2 / 3}
$$

and

$$
\nu_{\text {cool }} \propto \phi_{\mathrm{B}}^{-3} M^{-2} \propto \dot{m}^{-3 / 2} M^{-1 / 2}
$$

see Heinz \& Sunyaev (2003) and Heinz (2004).

This implies that, for sufficiently low accretion rates and high black hole masses, the radio emission from the jet will become optically thin. This must lead to a clear break in the scaling relation between $L_{\mathrm{r}}, L_{\mathrm{X}}$, and $M$.

Similarly, for sufficiently high accretion rates and high black hole masses, the X-ray emission from the jet will be affected by radiative cooling. In cases where the X-ray luminosity is dominated by the jet, this will lead to another break in the observed scaling relations. 
Finally, because the X-ray emission from the jet and from the accretion flow have different dependences on $\dot{m}$, another break in the scaling relations may occur from disk to jet X-rays at sufficiently low accretion rates and high masses. Whether such a break manifests itself in the existing samples of black holes depends on the unknown relative normalizations of the X-ray synchrotron luminosity and the inefficien disk X-ray luminosity.

The particular choice of the characteristic break and cooling frequencies, and the relative normalizations of jet and accretion luminosity, will determine where these respective breaks occur and whether any of them will affect the observed black hole samples.

In Fig. 1, we show the scaling indices in different regimes for three representative cases: One dominated by emission from the accretion flow, one dominated by emission from the jet, and a mixed case dominated by jet X-ray emission at low accretion rates and X-rays from the accretion flow at high accretion rates.

As Fig. 1 indicates, in the accretion dominated regime the range in scaling indeces is well represented by the fiducial values derived in Merloni, Heinz, \& di Matteo (2003), with exceptions only at very low accretion rates and large masses, where jets become optically thin in the radio. In the case of jet dominated X-rays, the adherence to a single scaling relation depends critically once again on the optical depth of the radio jet and on the location of the cooling break. Finally, in the more pathological case shown in the bottom panels, the scaling behavior is rather complex. Note that this case was constructed to show the largest variety of regimes.

This diversity in scaling indices might be interpreted as cause for concern. However, given the relatively steep scaling of X-ray luminosity with accretion rate for inefficient accretion and, to a lesser extent, for un-cooled optically thin synchrotron emission, a relatively narrow range of accretion rates is required to explain the observed spread in $\mathrm{X}$-ray luminosities in sample probing the fundamental plane. Thus, even in a mixed case as seen in the bottom figure, a large fraction of the sample will fall into a single scaling regime.

We discuss these possible breaks in scaling relations as a caveat to expecting a single realtion to hold for all black holes. Deviations should be expected in particular at low accretion rates and high masses, as evidenced by the figure.

It should also be noted that none of the above scaling relations make any specific assumptions about the geometry of the radio emitting region (i.e., conical vs. collimating vs. de-collimating jet). In fact, the relations are purely an expression of scale invariance and the radiative transfer of synchrotron radiation. As such, they must hold for any scale invariant flow that emits synchrotron radiation. The new "hot accretion" solution proposed by (2012)Veledina, Poutanen, \& Vurm (2012) falls into this regime, since it is (a) scale invariant, and (b) produces powerlaw synchrotron emission in the infrared (thus, the underlying scaling relation will be between infrared emission, mass, and accretion rate).

\section{Implications: New Radio-X-Ray Branches?}

Section 2 presents options for interpretations of any observed deviations from the classic scalings discovered in Gallo, Fender, \& Pooley (2003) and Merloni, Heinz, \& di Matteo (2003).

For example, the Radio-X-ray scalings for BL-Lacs can be understood from the properties of synchrotron spectra themselves, as already indicated by Plotkin, Markoff, Kelly, Körding, \& Anderson (2012). On the other hand, the fact that BL-Lacs do not deviate substantially from the fundamental plane for non-boosted sources suggests that X-ray 
synchrotron emission does not completely dominate the spectrum in un-boosted sources, since X-ray emission from jets is more strongly affected by Doppler boosting than radio emission (a simple consequence of the relativistic K-correction).

A particularly interesting question arises from the newly observed steeper branch of the radio-X-ray relation in XRBs [Coriat et al. (2011) and Gallo, Miller, \& Fender (2012)].
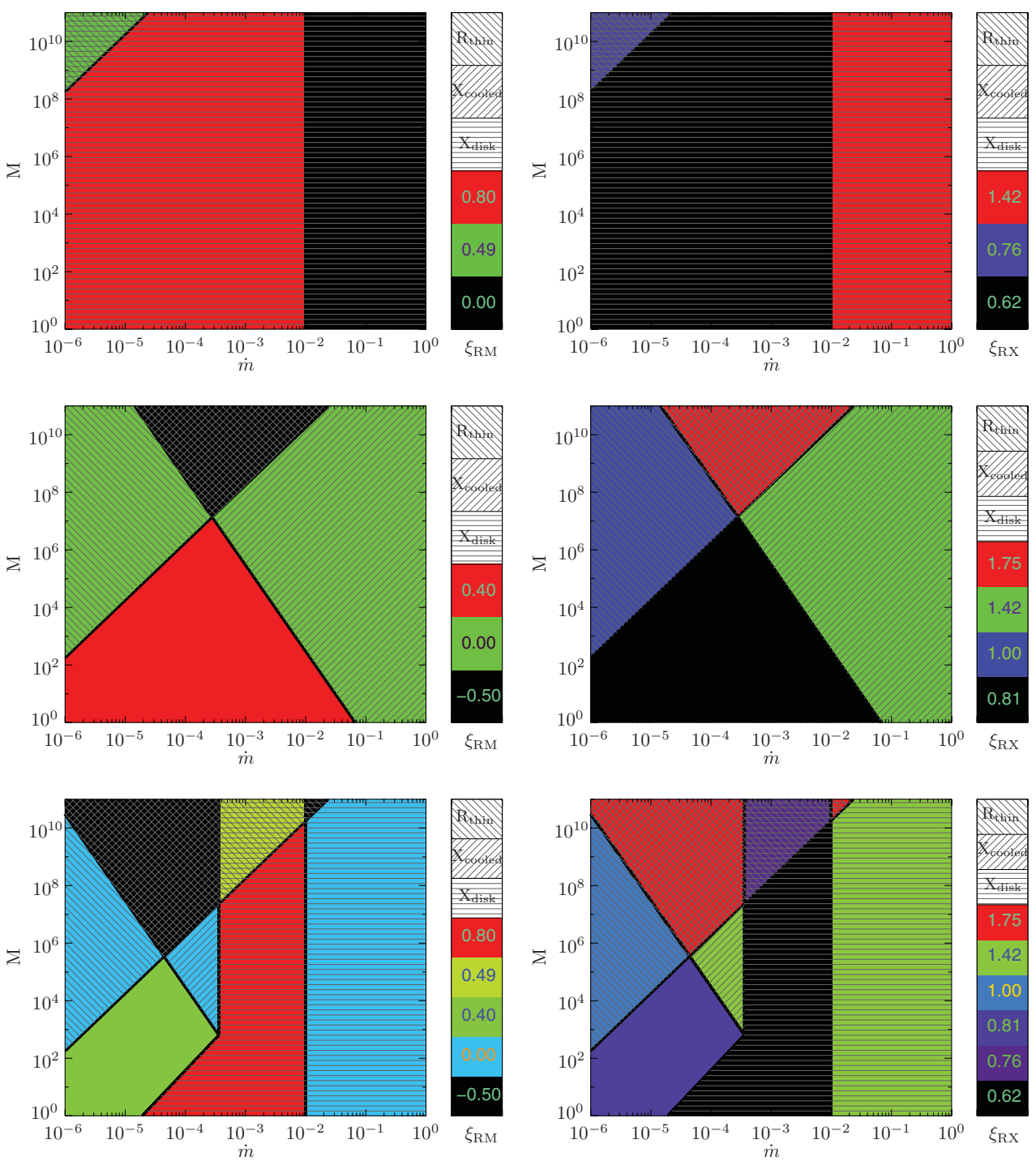

Figure 1. Scaling indeces $\xi_{\mathrm{RM}}$ between $L_{\mathrm{R}}$ and $M$ (left) and $\xi_{\mathrm{RX}}$ between $L_{\mathrm{R}}$ and $L_{\mathrm{X}}$ (right) for different regimes (indicated by hatching), with an assumed transition to efficient accretion at $\dot{m}=10^{-2}$, for accretion dominated X-ray emission (top), jet dominated X-ray emission (middle), and mixed X-ray emission (bottom). The region where X-ray emission is dominated by the accrtion flow is indicated by the horizontally hatched area; cases where the radio jet emission is optically thin are indicated by diagonally hatched shading (top-left to bottom-right) and regions where X-ray emission from the jet is dominated by cooled synchrotron emission is indicated by dianogally hatched shading (bottom-left to top-right). Note that in all cases, we have not accounted for the putative termination of the jet in the classic soft state above $\dot{m}_{\text {crits }}$. Illustrations of the resulting radio-X-ray relations for different black hole masses are given in 2 . 
This branch appears to originate at the transition from the soft to the hard state, at high accretion rates. Rather than descending down the typical radio-X-ray relation, which is characterized by a shallow dependence of $L_{\mathrm{R}} \propto L_{\mathrm{X}}^{0.7}$, some sources appear to follow a steeper track with $L_{\mathrm{R}} \propto L_{\mathrm{X}}^{1-2}$. The exact value of the slope is not yet well determined, but it is sufficiently different from the canonical value to allow some speculation about possible reasons.

It is clear from table 1 that several combinations predict a steep scaling: All efficient disk accretion solutions predict a scaling index $\xi_{\mathrm{RX}}$ significantly steeper than one. The same holds for jet emission affected by cooling. However, one would expect a soft X-ray spectrum in both cases, while the steep branch resides in the hard state [Gallo, Miller, \& Fender (2012)].

In addition to the separate scaling slope, it appears that the radio spectra of sources on the steep branch are systematically steeper. possibly indicating optically thin synchrotron emission in the radio. However, while optically thin synchrotron emission leads to generically steeper scaling relations between radio and X-ray emission, this difference is insufficient to explain the observed steepening without envoking a change in the behavior of the X-ray emission compared to the canonical hard state that leads to the canonical shallower radio-X-ray relation.

The other case consistent with this range of slopes is that of tically thin synchrotron emission for both radio and X-ray: In this case, the two components are connected by an unbroken powerlaw and must by definition track each other linearly, giving $\xi_{\mathrm{RX}}=1$. However, in this case the freedom in normalization between radio and X-ray emission
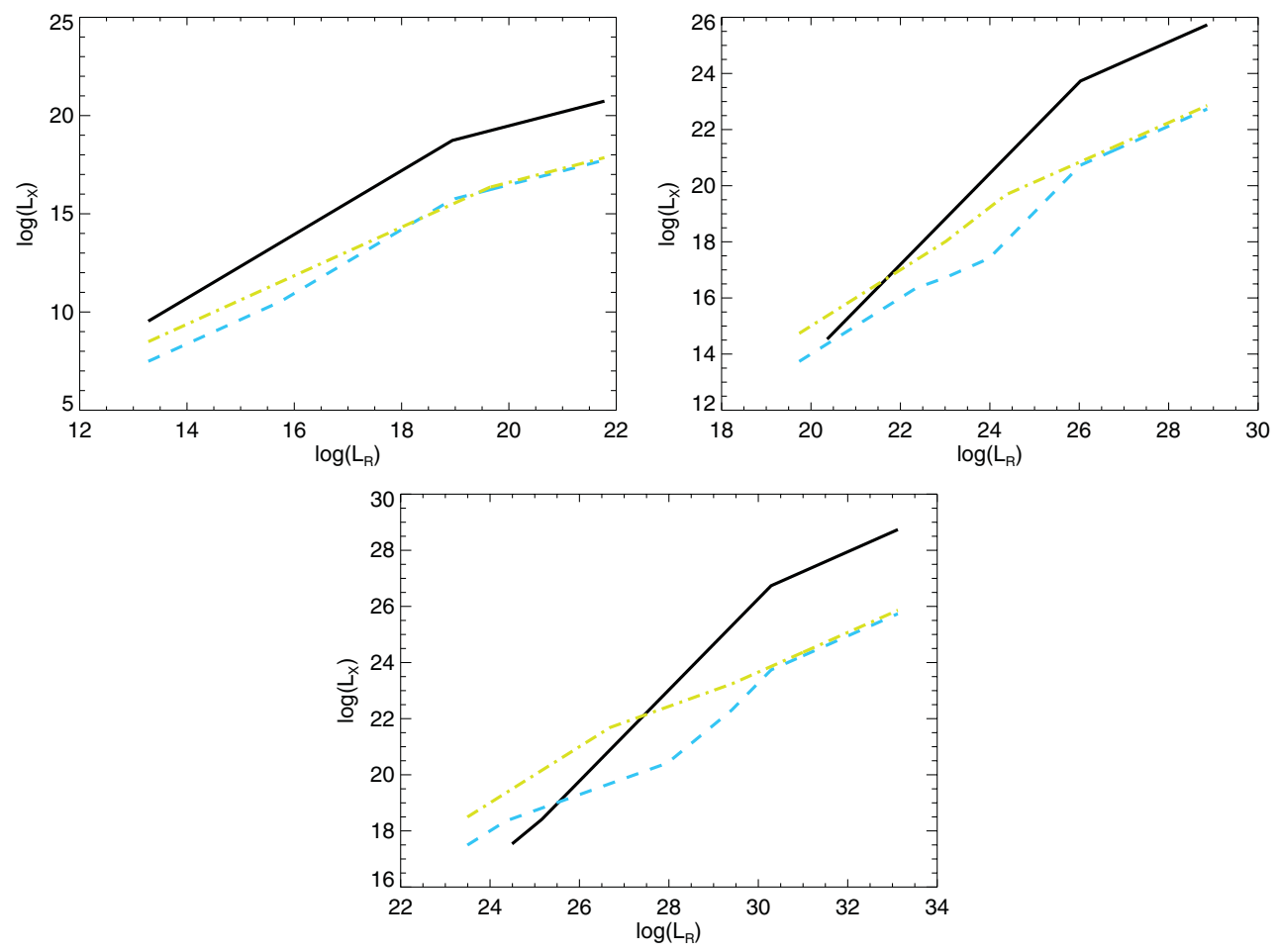

Figure 2. Illustrations of radio-X-ray relations for three different mass examples of $10 M_{\odot}$, $10^{6} M_{\odot}$, and $10^{9} M_{\odot}$ (top to bottom) for the three realizations of x-ray emission considered in Fig.1 (black solid: accretion dominated; yellow dash-dotted: jet dominated; cyan dashed: mixed). 
is removed, and the $\mathrm{X}$-ray luminosity $L_{\mathrm{X}}$ in terms of the radio luminosity $L_{\mathrm{R}}$ is simply given by $L_{\mathrm{X}}=L_{\mathrm{R}}\left(\nu_{\mathrm{R}} / \nu_{\mathrm{X}}\right)^{\alpha} \sim 10^{-4}$, which is about two orders of magnitude too small to explain the observed level of X-ray emission.

Given that the steep branch occurs at high X-ray luminosities (above $1 \%$ of the Eddington luminosity for a $10 M_{\odot}$ black hole), it is not implausible to suggest a difference in efficiency for hard-state accretion. A detailed discussion of X-ray states and spectral composition is, however, beyond the scope of this article.

Interestingly, sources on the steep branch appear to re-join the shallow branch at lower luminosities. The required slope for such a transition would be shallower than the canonical radio-X-ray slope. Because none of the alternative scaling indices are significantly shallower than the canonical value, and because of the fact that the $\mathrm{X}$-ray luminosity is only a proxi for the accretion rate, this suggests that the underlying mechanism of the $\mathrm{X}$-ray emission changes during this transition.

Should the second branch solidify into a significantly steeper, robust radio-X-ray relation, joined to a significantly shallower part, interesting conclusions about the radiative efficiency of X-ray emission from the hard state could be drawn.

\section{References}

Falcke, H., Körding, E., \& Markoff, S. 2004, A\&A, 414, 895

Broderick, J. W. \& Fender, R. P. 2011, MNRAS, 417, 184

Coriat, M., Corbel, S., Prat, L., et al. 2011, MNRAS, 414, 677

Gallo, E., Fender, R. P., \& Pooley, G. G. 2003, MNRAS, 344, 60

Gallo, E., Miller, B. P., \& Fender, R. 2012, MNRAS, 423, 590

Heinz, S. 2004, MNRAS, 355, 835

Heinz, S. \& Merloni, A. 2004, MNRAS, 355, L1

Heinz, S. \& Sunyaev, R. A. 2003, MNRAS, 343, L59

Merloni, A., Heinz, S., \& di Matteo, T. 2003, MNRAS, 345, 1057

Plotkin, R. M., Markoff, S., Kelly, B. C., Körding, E., \& Anderson, S. F. 2012, MNRAS, 419, 267

Veledina, A., Poutanen, J., \& Vurm, I. 2012, ArXiv e-prints

Yuan, F., Yu, Z., \& Ho, L. C. 2009, ApJ, 703, 1034 